\title{
Correction to \\ Fundamental theorem for the projective line over non-commutative local rings
}

\author{
By
}

B. V. Lmaye and N. B. Lmaye

The following should replace lines 4 and 5 on page 104 of Volume 28 (1977):

Let $L$ be a ring with 1 , and $a, b$ and $k$ be units in $L$. If $1-k a b$ is also a unit, then

$$
a b a=k^{-1}\left\{a-\left\{a^{-1}+k\left(b^{-1}-k a\right)^{-1}\right\}^{-1}\right\}
$$

if and only if $k$ commutes with $a$.

Eingegangen am 9. 8.1977 